\title{
A Dynamically Grouped Multi-multicast Stream Scheduling Strategy for Video-on-Demand Systems"
}

\author{
Dafu Deng, Hai Jin, and Zongfen Han \\ Huazhong University of Science and Technology, Wuhan, 430074, China \\ hjin@hust.edu.cn
}

\begin{abstract}
Network bandwidth is the key performance bottleneck for a videoon-demand $(\mathrm{VoD})$ server. It controls the number of clients the server can support simultaneously. Previous works have shown some strategies, such as the batching strategy and the stream merging strategy, that use one multicast stream to serve different clients requesting the same video object at the same time. They improve the performance of server bandwidth effectively. But the batching strategy results in long start-up latency and the traditional stream merging strategy also wastes lots of server bandwidth. In this paper, we propose a $d y$ namically grouped multi-multicast stream scheduling strategy, called DGMM, and analyze its performance in two factors: the start-up latency and the average bandwidth consumption.
\end{abstract}

\section{Introduction}

Video-on-Demand ( $\mathrm{VoD})$ is a service that allows clients to communicate with a video server selecting and viewing a video of their choice simultaneously. A few companies, such as IBM, Microsoft and Apple, successfully ran VoD systems serving a lot of clients with high cost. With broadband network technology, the client-end bandwidth can achieve almost 100Mbits/s. It can support up to 60 MPEG-I streams (approximately $1.5 \mathrm{Mbits} / \mathrm{s}$ per MPEG-1 stream). Therefore, server I/O bandwidth and network bandwidth are the major limiting factors in the widespread usage of $\mathrm{VoD}$ systems. They control the number of streams concurrently sent by a video server.

A conventional video server simply schedules one requested stream for each client, while most clients often request the same hot video at the same time [5][6]. This phenomenon results in that conventional VoD servers send some streams of same video data at the same time. It wastes a mass of server bandwidth. Still conventional systems do not scale well, and better solutions are necessary.

The batching strategy $[1] \mid 5] \mid[6 \mid$ 9 $]$ presents one such solution. It uses a single multicast stream of data to serve clients that request the same hot video during the same short time interval. In the batching strategy, a time threshold must be set firstly. Video servers just schedule the multicast stream at the end of each time threshold. In order to obtaining a good efficiency in usage of bandwidth, value of this time threshold must be at least 7 minutes [5] Therefore, the expected start-up latency is approximate 3.5 minutes. This long delay increases clients reneging rate and decreases the popularization of $\mathrm{VoD}$ systems.

\footnotetext{
* This paper is supported by Wuhan Key Hi-Tech Project under grant 20011001001.
} 


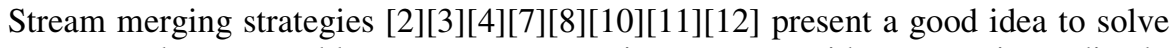
long start-up latency problem. In stream merging strategy, video server immediately schedules one or two kinds of streams to serve each client request: the complete multicast stream and the patching unicast stream. For the first arrived request, the video server schedules a complete multicast stream to transmit the integrated video object immediately. For later requests with the same object, the video server firstly notifies them to join the multicast group for receiving the remainder of scheduled complete multicast stream, and then, schedules a patching multicast stream to transmit the lost video data. Thus, later starters must concurrently receive two streams and merging them into an integrated video stream. This strategy guarantees the zero start-up latency and high server bandwidth efficiency when client request rates are low and moderate. However, the server bandwidth efficiency is decreased dramatically at high request rates due to mass retransmission of same video data.

This paper contributes in developing a novel stream scheduling strategy, called Dynamically Grouped Multi-Multicast, which can significantly improving server bandwidth efficiency over a wide range of client request rates. The subsequent sections are organized as follows. In section 2 , we describe the basic idea of dynamically grouped multi-multicast stream strategy, and in section 3, introduce an example algorithm. Section 4 presents performance evaluation based on experimental research. Finally, section 5 contains our concluding remarks and future works.

\section{Dynamically Grouped Multi-multicast Scheduling Strategy}

\subsection{Preliminaries}

Consider that clients requesting different hot video objects are served independently. Clients request the same hot video object will be discussed in the following sections. Time is divided into fixed size intervals $T$. The time slot in which the first client request arrived is labeled $t_{0}$. The following time slots are labeled $t_{1}, \ldots, t_{n}(0 n<+\infty)$. The hot video with $\mathrm{L}$ minutes is divided into $\lceil\mathrm{L} / \mathrm{T}\rceil$ fixed size segments, denoted as $\mathrm{V}_{0}, \mathrm{~V}_{1}$, $\ldots, \mathrm{V}_{\Gamma_{\mathrm{L} T} \mathrm{~T}_{-1}}$. Other conceptions are defined as follows.

Clients requests: For requests arriving at the same time slot, video servers can batch them together and serve them all by a multicast stream. Therefore, those requests are considered as one request. We use $\mathrm{C}_{\mathrm{i}}$ to represent the request arriving at the time slot $\mathrm{t}_{\mathrm{i}}$.

Requests group: Given a clients' requests sequence $C_{0}, \ldots, C_{i}, \ldots, C_{j}, \ldots, C_{n}$ $(0<\mathrm{i}<\mathrm{j}<\mathrm{n}<+\infty)$, the sequence is not continuous because requests may not arrive in some time slots. We sequentially divide those requests into several flexible sized requests groups $\mathrm{G}_{0}, \mathrm{G}_{1}, \ldots, \mathrm{G}_{\mathrm{k}}(0<\mathrm{k}<+\infty)$, while $\mathrm{G}_{\mathrm{k}}$ is called the $\mathrm{k}$-th requests group.

Complete multicast stream (CS): For requests group $\mathrm{G}_{\mathrm{i}}$, in order that all clients can receive all the video segments, video servers must schedule a multicast stream, which transmits all the segments. This multicast stream is defined to be the complete multicast stream. We use $\mathrm{CS}_{\mathrm{i}}$ to represent the complete multicast stream in requests group $\mathrm{G}_{\mathrm{i}}, \mathrm{N}_{\mathrm{cs}}(\mathrm{i})$ represents the serial number of the time slot at the end of which $\mathrm{CS}_{\mathrm{i}}$ is scheduled, and $\ell\left(\mathrm{CS}_{\mathrm{i}}\right)$ represents the number of video segments transmitted on $\mathrm{CS}_{\mathrm{i}}$. 
Patching multicast stream (PS): In a requests group $\mathrm{G}_{\mathrm{i}}$, once $\mathrm{CS}_{\mathrm{i}}$ has been initialized, clients with requests arrival time later than time $t_{\mathrm{Ncs}(\mathrm{i})}$ may miss some video segments that have been transmitted. Therefore, video server must initialize several streams to patch the lost video segments to clients. We use patching multicast streams to define those streams and use $\operatorname{PS}_{\mathrm{k}}(0<\mathrm{k} \leq+\infty)$ to present the patching multicast stream initialized at the end of time slot $t_{k} \cdot \ell\left(\mathrm{PS}_{\mathrm{k}}\right)$ is used to represent the number of video segments transmitted on $\mathrm{PS}_{\mathrm{k}}$.

Segment transmission time (STT): Assuming that video server use CBR (constant bit rate) transmission mode, any segment must be transmitted completely in a time slot. Therefore, if a video segment is transmitted on a stream, the transmission must begin at a fixed time point, called segment transmission time (STT). We use $\Gamma(\mathrm{m}, \mathrm{n})$ to represent the segment transmission time of segment $\mathrm{V}_{\mathrm{m}}$ transmitted on stream $\mathrm{PS}_{\mathrm{n}}$. The following formula can be deduced: $\Gamma(\mathrm{m}, \mathrm{n})=\mathrm{t}_{\mathrm{n}}+\mathrm{m}^{*} \mathrm{~T}$.

\subsection{Basic Idea of DGMM}

DGMM scheduling strategy determines how to divide the requests sequence into different requests groups and how to schedule streams for intra-group requests and inter-group requests so that the bandwidth efficiency can be improved significantly. Rules of DGMM strategy are described as follows.

Grouping rule: A video server dynamically maintains several request groups $\mathrm{G}_{0}$, $\mathrm{G}_{1}, \ldots, \mathrm{G}_{\mathrm{n}}$. When the first client request arrived, the video server creates requests group $\mathrm{G}_{0}$ and puts the request $\mathrm{C}_{0}$ into $\mathrm{G}_{0}$. For other request $\mathrm{C}_{\mathrm{i}}$, if $\mathrm{t}_{\mathrm{i}}<\mathrm{t}_{\mathrm{cs}}+(\lceil\mathrm{L} / \mathrm{T}\rceil-1) * \mathrm{~T}$, where $\mathrm{t}_{\mathrm{cs}}$ is the first request arrival time slot of the latest created requests group $G_{n}$, the video server puts the request into $G_{n}$. Otherwise, it creates a new requests group $G_{n+1}$ and puts the request into it. When all requests of the requests group $\mathrm{G}_{\mathrm{i}}$ have been served, $\mathrm{G}_{\mathrm{i}}$ is deleted. For example, as shown in Figure 1 , requests $\mathrm{C}_{0}, \mathrm{C}_{1}, \mathrm{C}_{2}, \mathrm{C}_{3}, \mathrm{C}_{4}, \mathrm{C}_{5}, \mathrm{C}_{6}, \mathrm{C}_{7}$ are put into requests group $\mathrm{G}_{0}$ because their requests arrival time slots are less than $\mathrm{t}_{0}+(\lceil\mathrm{L} / \mathrm{T}\rceil-1)^{*} \mathrm{~T}$. Request $\mathrm{C}_{14}$ is grouped into $\mathrm{G}_{1}$ because time slot $\mathrm{t}_{14}$ is later than $\mathrm{t}_{0}+(\lceil\mathrm{L} / \mathrm{T}\rceil-1) * \mathrm{~T}$.

Inter-group rule: According to the above grouping rule, there is no video segments can be shared among inter-group requests. Therefore, the video server handles each grouped requests independently.

Intra-group rule: The objective of each client request in a requests group $\mathrm{G}_{\mathrm{i}}$ is to receive all the $\lceil\mathrm{L} / \mathrm{T}\rceil$ parts of transmission and view them without any interruption. For the first client request of requests group $G_{i}$, the video server schedules a complete multicast stream $\mathrm{CS}_{\mathrm{i}}$ at the end of the first request arrival time slot. The corresponding clients join the multicast group of $\mathrm{CS}_{\mathrm{i}}$ to receive and then playback all segments of requested videos. For any other request $\mathrm{C}_{\mathrm{k}}\left(\mathrm{N}_{\mathrm{cs}}(\mathrm{i})<\mathrm{k}<\mathrm{N}_{\mathrm{cs}}(\mathrm{i})+[\mathrm{L} / \mathrm{T}\rceil-1\right)$, as video segments $\mathrm{V}_{0}, \ldots, \mathrm{V}_{\mathrm{k}-\mathrm{Ncs}(\mathrm{i})-1}$ have been transmitted, the video server firstly notifies the corresponding clients to join the multicast group of $\mathrm{CS}_{\mathrm{i}}$ to receive and buffer video segments $\mathrm{V}_{\mathrm{k} \text {-Ncs(i) }}, \ldots, \mathrm{V}_{\left.\Gamma_{\mathrm{L} / \mathrm{T}}\right\rceil_{-1}}$. Then, for each segment of $\mathrm{V}_{0}, \ldots, \mathrm{V}_{\mathrm{k} \text {-Ncs(i)-1 }}$, the video server searches all the existed patching multicast streams $\mathrm{PS}_{\mathrm{Ncs(i)+1}}, \ldots, \mathrm{PS}_{\mathrm{k}-1}$.

If there are no patching multicast streams existed, it schedules a patching multicast stream $\mathrm{PS}_{\mathrm{k}}$ to transmit video segments $\mathrm{V}_{0}, \ldots, \mathrm{V}_{\mathrm{k}-\mathrm{Ncs}(\mathrm{i})-1}$ and notifies the corresponding clients to receive and playback those video segments. If video segment $\mathrm{V}_{\mathrm{j}}(0 \leq \mathrm{j} \leq \mathrm{k}-$ 
$\left.\mathrm{N}_{\mathrm{cs}}(\mathrm{i})-1\right)$ is transmitted on a existed patching multicast stream $\operatorname{PS}_{\mathrm{m}}\left(\mathrm{N}_{\mathrm{cs}}(\mathrm{i})<\mathrm{m}<\mathrm{k}-\mathrm{N}_{\mathrm{cs}}(\mathrm{i})\right)$ and the transmit time $\Gamma(\mathrm{j}, \mathrm{m}) \geq \mathrm{t}_{\mathrm{k}}$, the video server notifies those clients to join the multicast group of $\mathrm{PS}_{\mathrm{m}}$ to receive segment $\mathrm{V}_{\mathrm{j}}$. At last, for video segments not transmitted on existed streams or the segment transmission time of them is less than $t_{k}$, the video server schedules a patching multicast stream $\mathrm{PS}_{\mathrm{k}}$ at the end of time slot $\mathrm{t}_{\mathrm{k}}$ and transmits them at the corresponding segment transmission time. The corresponding clients $\mathrm{C}_{\mathrm{k}}$ must also join the multicast group of $\mathrm{PS}_{\mathrm{k}}$ to receive those segments.

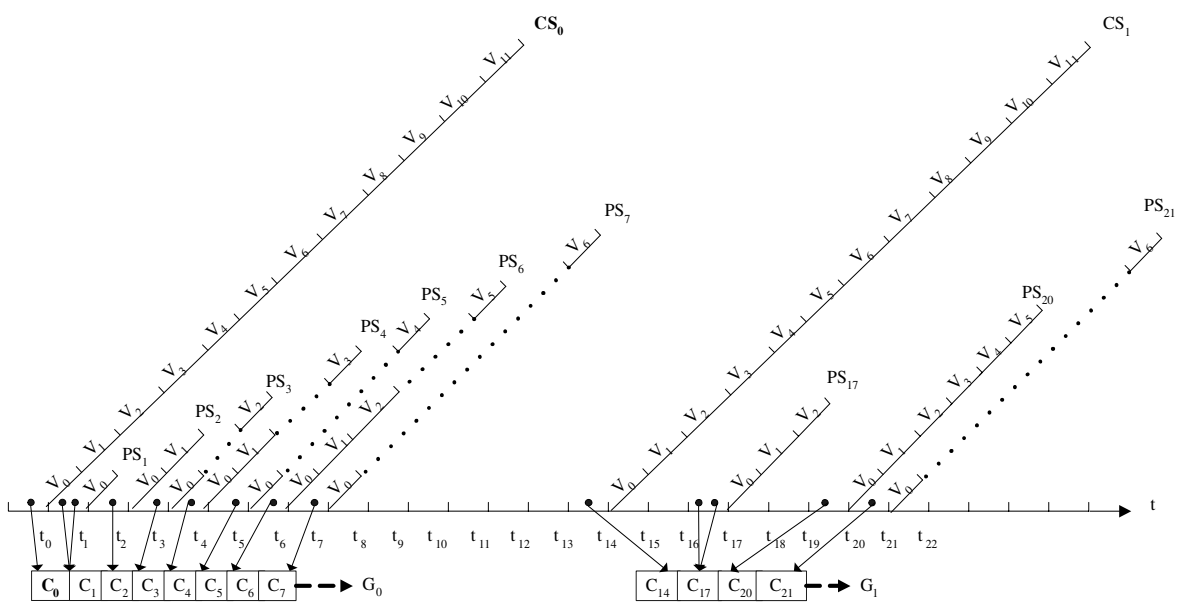

Fig. 1. A scheduling example of DGMM

Figure 1 shows a scheduling example of DGMM. The solid lines describe multicast streams, while the dotted lines indicate the skipped video segments. Because it is similar between requests group $\mathrm{G}_{0}$ and requests group $\mathrm{G}_{1}$, we just discuss the scheduling for group $\mathrm{G}_{1}$. CS $\mathrm{S}_{1}$ is the complete scheduled multicast stream and the client request $\mathrm{C}_{14}$ joins the multicast group of $\mathrm{CS}_{1}$ to receive and playback all segments. Because segments $\mathrm{V}_{0}, \mathrm{~V}_{1}, \mathrm{~V}_{2}$ have been transmitted completely when request $\mathrm{C}_{17}$ arrives, the video server schedules $\mathrm{PS}_{17}$ to transmit those missed segments. The client $\mathrm{C}_{17}$ join the multicast group of $\mathrm{CS}_{1}$ to receive and buffer segments $\mathrm{V}_{3}, \ldots, \mathrm{V}_{11}$ and join the multicast group of $\mathrm{PS}_{17}$ to receive and playback segments $\mathrm{V}_{0}, \mathrm{~V}_{1}, \mathrm{~V}_{2}$.

The similar case is happened for request $\mathrm{C}_{20}$. For serving the request $\mathrm{C}_{21}$, the video server firstly notifies the corresponding clients to join the multicast group of $\mathrm{CS}_{1}$ to receive and buffer segments $\mathrm{V}_{7}, \mathrm{~V}_{8}, \mathrm{~V}_{9}, \mathrm{~V}_{10}, \mathrm{~V}_{11}$. Then, it searches the existed patching multicast streams $\mathrm{P}_{17}, \mathrm{P}_{18}$ and finds that segments $\mathrm{V}_{1}, \mathrm{~V}_{2}, \mathrm{~V}_{3}, \mathrm{~V}_{4}, \mathrm{~V}_{5}$ can be received on PS $_{18}$ by client $C_{21}$ (i.e. $\left.\Gamma(1,18), \ldots, \Gamma(5,18) \geq t_{21}\right)$. Therefore, the server notifies $C_{21}$ to join the multicast group of $\mathrm{PS}_{18}$ to receive those segments. At last, the server schedules a patching multicast stream $\mathrm{P}_{21}$ to transmit segments $\mathrm{V}_{0}, \mathrm{~V}_{6}$ at the corresponding segment transmission time. The client $\mathrm{C}_{21}$ also needs to join the multicast group of $\mathrm{PS}_{21}$ to receive segments $\mathrm{V}_{0}, \mathrm{~V}_{6}$. 


\section{An Example Algorithm of DGMM}

A video server is usually comprised of three parts--scheduler, data server, and video storage. A scheduler is a controller of the video server. It is responsibility for exchanging control messages with clients on a control channel and for scheduling a data server to transmit video segments. Functions of a data server are reading video segments from a video storage and allocating data channels to transmit video segments. When a client request arrives, the scheduler determines data channels a client should join and sends a message list $\mathrm{M}_{1}$ to notify the information of those channels. The information of each data channel is embedded in one element of $\mathrm{M}_{1}$. We use a triple $\mathrm{M}_{\mathrm{i}}\left(\mathrm{a}, \mathrm{A}, \mathrm{S}_{\mathrm{Id}}\right)$ to describe the $\mathrm{i}$-th element of $\mathrm{M}_{1}$, where $a$ is the multicast IP address of that channel, $A$ is a bit array indicating which video segment is transmitted on that channel, and $\mathrm{S}_{\mathrm{Id}}$ is the exclusive identity of that channel. Here we discuss an example algorithm for the client, the scheduler, and the data server.

\subsection{Client Algorithm}

Each client contains a set-top box, a disk, and a display monitor. Clients communicate with a video server by the set-top box. There is a controlling thread, a network listening thread, a task thread, a display thread, and a buffer management thread running in the set-top box. The controlling thread requests its desired video, waits to receive message list $\mathrm{M}_{1}$ comprised of $\mathrm{M}_{0}\left(\mathrm{a}, \mathrm{A}, \mathrm{S}_{\mathrm{Id}}\right), \ldots, \mathrm{M}_{\mathrm{i}}\left(\mathrm{a}, \mathrm{A}, \mathrm{S}_{\mathrm{Id}}\right)$. For each message, the controlling thread notifies the network listening thread to listen in the corresponding data channel. When a video segment arrives on a multicast stream, the network listening thread notifies the task thread to run the corresponding task to receive them. Each task is responsible for receiving video segments on one data channel, and sending them to the display thread or save them to disk. Figure 2(a) shows the client data receiving algorithm in detail.

\subsection{Server Scheduler Algorithm}

The key function of a scheduler is to determine in which data channel a client can receive valid video segments and how many video segments should be transmitted on that channel. In order to implementing this function, a stream information list is built for all data channels allocated to serve a group requests. Each unit of the stream information list describes the information of one data channel in a triple $\mathrm{S}_{\mathrm{i}}(\mathrm{t}, \mathrm{a}, \mathrm{A})$, where $t$ is the initiating time of a data channel, $a$ is the multicast IP address of the data channel, and $A$ is an array with each element the serial number of a video segment transmitted on data channel. Furthermore, a message list $\mathrm{M}_{1}$ comprised of $\mathrm{M}_{0}(\mathrm{a}, \mathrm{A}$, $\left.\mathrm{S}_{\mathrm{Id}}\right), \ldots, \mathrm{M}_{\mathrm{i}}\left(\mathrm{a}, \mathrm{A}, \mathrm{S}_{\mathrm{Id}}\right)$ is built to record the information of data channels allocated for one request. The video server uses this message list to notify that client to receive video segments on the corresponding channels.

Figure 2(b) illustrates the server scheduler algorithm. The scheduling thread is driven by two kinds of events: the request arrival event and the time out event. The request arrival event is started when a request arrives, while the time out event is started at the end of each time slot. Each event includes a parameter to indicate the 
requested video object so that the scheduler can find out appropriate scheduling parameters.

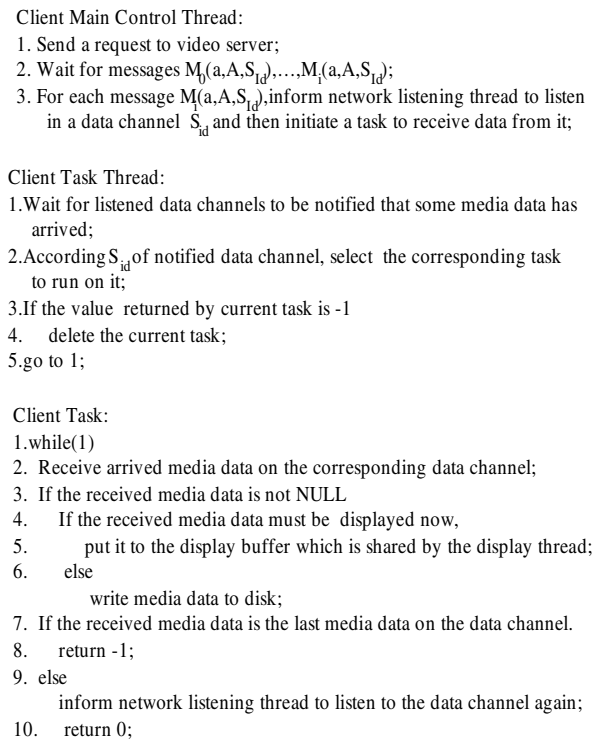

(a)Client receiving algorithm

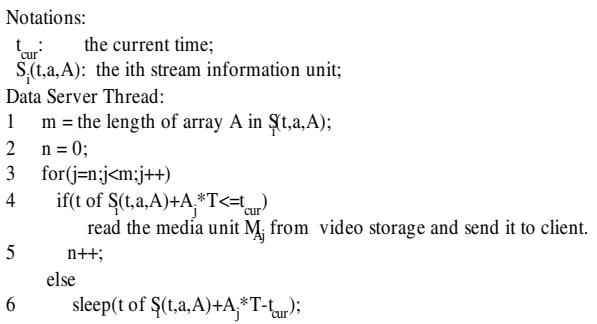

(c)Data server algorithm

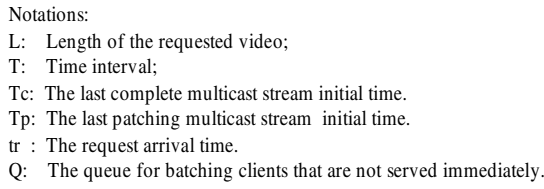

L: Length of the requested video;

$\mathrm{T}$ : Time interval;

Tc: The last complete multicast stream initial time.

$\mathrm{Tp}$ : The last patching multicast stream initial time.

tr : The request arrival time.

Q: The queue for batching clients that are not served immediately.

Scheduler Thread Algorithm:

1 If a request arrival event start

2 Finds out corresponding parameters Q,Tc, and $\mathrm{L}$ based on requested video object.

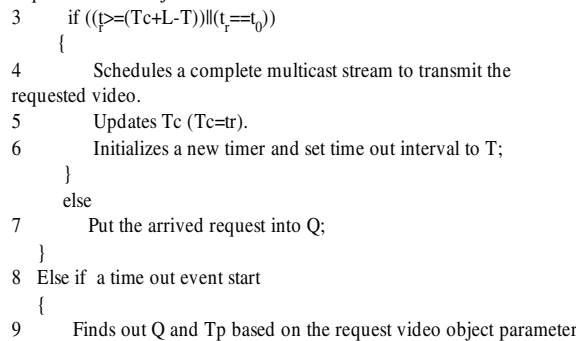

(b) Server scheduler algorithm

Fig. 2. An example algorithm of DGMM.

\subsection{Data Server Algorithm}

Each data server thread is responsible for serving a multicast stream. The function of a data server thread is reading media data from a video storage, and then sending them to clients on a data channel according to the corresponding stream information unit $\mathrm{S}_{\mathrm{i}}(\mathrm{t}, \mathrm{a}, \mathrm{A})$. On a special data channel, a video segment must be transmitted at a fixed time point, and the multicast stream may be not continual. Therefore, we must interrupt the data server thread and wake up it at the appropriate time point. Figure 2(c) shows the data server algorithm. 


\section{Performance Evaluations}

In this section we will give out experimental results with comparison with the FCFS batching strategy and the stream merging strategy.

\subsection{Experimental Parameters}

We consider two factors for each video: its length and popularity. The data from Internet Movie Database (http://www.imdb.com) has shown a normal distribution with a mean of 102 minutes and a standard deviation of 16 minutes. In order to reserve some client bandwidth used to obtain other services (i.e FTP, WWW, Email), we assume that the maximum bandwidth for a client is 30 concurrent MPEG-1 streams (near $45 \mathrm{Mb} / \mathrm{s}$ ), and a video is split into several parts (usually 2 parts). The length of each part is less than 60 minutes, and each part is considered as an integrated video. Clients who request a video longer than 60 minutes must send request more than once. The time interval $T$ for each request is 1 minute. Therefore, a video is divided into 45 60 fixed size segments.

The popularity of each video was modeled using a Zipf-like distribution with parameter 0.271 . This is the mostly used distribution for VoD studies. In our experiment, the number of videos stored on a video storage is 100 . Client requests are generated using a Poisson arrival process with an interval time of $1 / \lambda$, for varying $\lambda$ values, between 100 to 1400 arrivals per hour. Clients simply selected a video, waited for their request to be served, and then watched the video until it was completed.

Video server and network are main components in VoD systems. For a video server, we utilize Darwin-streaming server (http://www.apple.com), which can support 600-800 MPEG-1 unicast streams simultaneously. We also use 1000M Ethernet to simulate the broadband network. Because the bandwidth for each NIC is $100 \mathrm{Mb} / \mathrm{s}$. Figure 3 shows the main experimental environment parameters.

\begin{tabular}{|l|l|}
\hline Video length (minutes) L & $45 \sim 60$ \\
\hline Number of videos Nv & 100 \\
\hline Video Format & M PE G -I \\
\hline Clients'maxim um bandwidth (M bits/s) & 100 \\
\hline Server's maxim um bandwidth(M bits/s) & 1000 \\
\hline Clients arrival rate $\boldsymbol{\lambda}(/$ hour) & $100 \sim 1400$ \\
\hline
\end{tabular}

Fig. 3. Experiment Parameters

\subsection{Results}

For a video server, there are two most important performance factors: start-up latency, which is the amount of time clients must wait to watch the chosen videos; and average bandwidth consumption, which indicates the bandwidth efficiency of a video server. 


\subsubsection{Start-up Latency}

Figure 4 displays the system average start-up latency at client request rates 100, 500, 800,1400 per hour, and shows how these latencies are affected by the number of served clients. We choose 100 to be the low request arrival rate, 500 and 800 to be the middle request arrival rate, and 1400 to be the high request arrival rate. As one can see, the system average start-up latency is changed very small under different request arrival rate. It always remains on $25 \pm 5$ seconds when there are enough clients' requests arrived. This latency can be accepted by almost all clients.

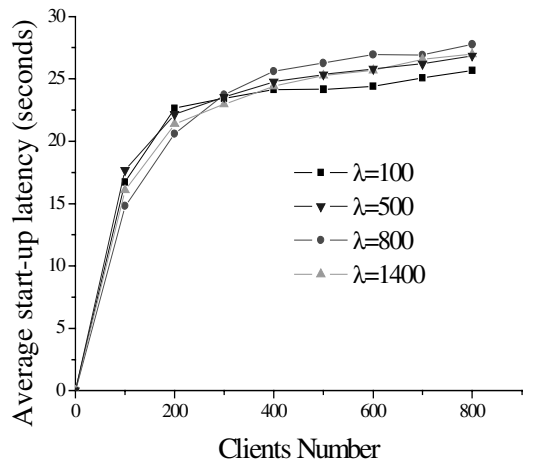

Fig. 4. Request arrival rate vs. average the start-up latency.

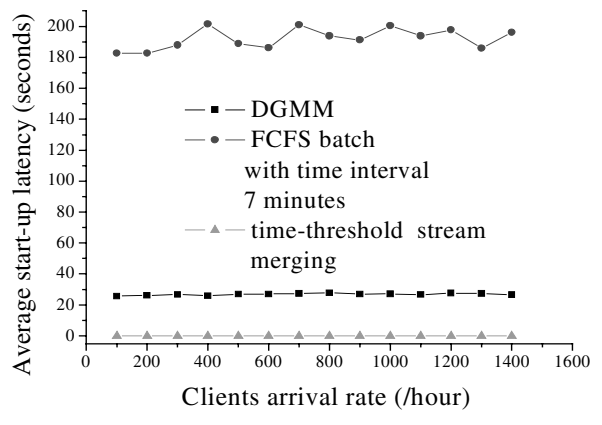

Fig. 5. A start-up latency comparison among FCFS batching strategy with the time interval 7 minutes, the time-threshold stream merging strategy with parameter $\mathrm{W}=20$ minutes and unconstrained clients buffer, and the DGMM strategy with time slot $=1$ minute

Figure 5 displays the start-up latency comparison among FCFS batching with time interval $\mathrm{T}=7$ minutes, the stream merging strategy with parameter $\mathrm{W}=20$ minutes and unconstrained client disk space and our DGMM. We choose 7 minutes because paper [5] have presented that FCFS batching could obtain a good tradeoff between start-up latency and bandwidth efficiency at this time interval. The same as to the stream merging strategy, paper [8] have proposed a time-threshold stream merging strategy and showed that the total performance was better than other traditional stream merging strategies when the time-threshold $W$ was 20 minutes and the client storage disk was unconstrained. As one can see, DGMM strategy outperforms the FCFS strategy and just is little poorer than the time-threshold stream merging strategy in the aspect of the system average start-up latency. The reason of little poor performance compared with the time-threshold stream merging strategy is that DGMM batches clients' requests arrived at the same time slot. This will increase the bandwidth efficiency.

\subsubsection{Bandwidth Consumption}

Figure 6 shows how request arrival rate affects the average server bandwidth consumption. We can find out that the average server bandwidth consumption is increased in some degree with the increasing of request arrival rate. The reason is that 
server will support more clients at a period of time. As showed on Figure 7, when request arrival rate is less than 150 requests per hour, the bandwidth consumption of three kinds of scheduling strategies are held in same level. But by the increasing of request arrival rate, the increasing degree of DGMM is distinctly less than FCFS batching and the time-threshold stream merging strategy. When request arrival rate is 500 , the average bandwidth consumption of DGMM is approximate $170 \mathrm{Mb} / \mathrm{s}$.

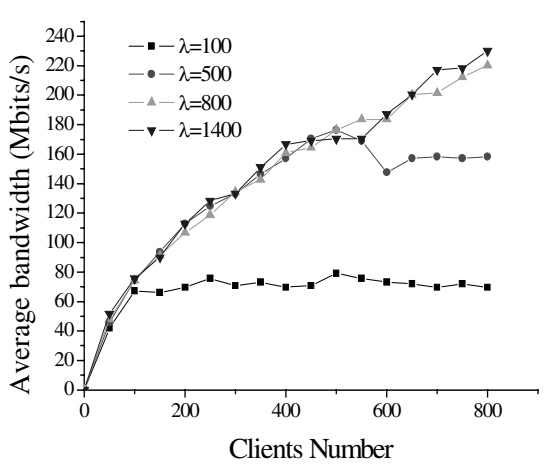

Fig. 6. Request arrival rate vs. average Bandwidth.

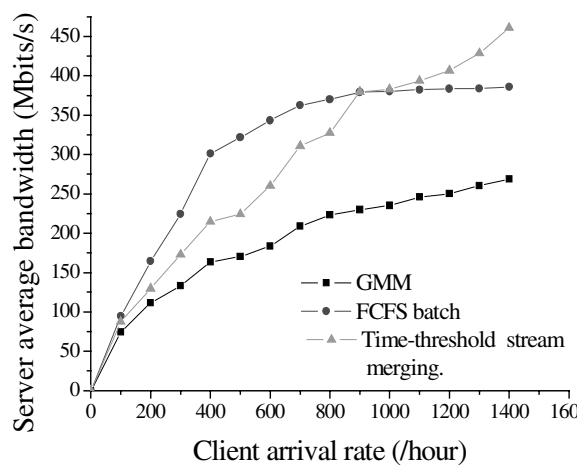

Fig. 7. An average bandwidth comparison among the FCFS batching strategy with time interval 7 minutes, the time-threshold stream merging strategy with parameter $\mathrm{W}=20 \mathrm{~min}-$ utes and unconstrained clients buffer, and the DGMM strategy with time slot $=1$ minute

At the same request arrival rate, the average bandwidth consumption of the FCFS batching is approximate $320 \mathrm{Mb} / \mathrm{s}$ and that of the time-threshold stream merging strategy is approximate $220 \mathrm{Mb} / \mathrm{s}$. At middle request arrival rate, DGMM can save approximate $45 \%$ and $23 \%$ bandwidth consumption compared with FCFS batching and time-threshold stream merging strategy, respectively. When request arrival rate is higher than 1000 requests per hour, the bandwidth performance of the time-threshold stream merging strategy is decreased dramatically. It is worse than FCFS batching. In any case, they are all worse than DGMM. (FCFS: approximate $380 \mathrm{Mb} / \mathrm{s}$; Timethreshold stream merging: approximate $400 \mathrm{Mb} / \mathrm{s}$; DGMM: $240 \mathrm{Mb} / \mathrm{s}$; at request rate: 1100 request per hour). Therefore, DGMM outperforms the FCFS batching strategy and the stream merging strategy at the aspect of bandwidth performance.

\section{Conclusions and Future Works}

This paper presents a novel stream scheduling strategy that significantly reduces the demand on the server network-I/O bandwidth. Unlike existing batching strategy and stream merging strategy, DGMM dynamically groups the clients' requests according to the request arrival time and schedules two kinds of multicast stream: continuous completely multicast stream and no-continuous patching multicast stream. This guarantees that no redundant video data are transmitted at the same time and the transmitting video data are shared among grouped clients. Furthermore, we give out an exam- 
ple algorithm and study the performance of DGMM from start-up latency and bandwidth consumption.

Time interval $T$ is an important issue in DGMM. It determines the number of streams that can be concurrently received by a client. If $T$ is too small, the number of concurrently received streams may be increased dramatically. Therefore, the bandwidth of clients may be exhausted. But if $T$ is too large, the start-up latency of clients may be too long to be endured. In this paper, we simply choose 1 minute to be the value of $T$ because most clients can accept the start-up latency less than 1 minute and current broadband network technology improves the bandwidth of client to $100 \mathrm{Mb} / \mathrm{s}$. In the near future, we will study the effects of time interval $T$ and propose an optimal time interval $T$.

\section{References}

[1] C. C. Aggarwal, J. L. Wolf, and P. S. Yu, "The Maximum Factor Queue Length Batching Scheme for Video-on-Demand Systems", IEEE Transactions on Computers, Vol.50, No.2, pp.97-110, Feb. 2001.

[2] S. W. Cater and D. D. E. Long, "Improving video-on-demand server efficiency through stream tapping", Proc. of ICCCN, Las Vegas, NV, Sept. 1997, pp.200-207.

[3] S. W. Cater and D. D. E. Long, "Improving bandwidth efficiency on video-on-demand servers", Computer Networks, Vol.30, No.1-2, pp.99-111, Jan. 1999.

[4] S.-H. G. Chan and E. Chang, "Providing scalable on-demand interactive video services by means of client buffering", Proc. IEEE ICC, Helsinki, Finland, June 2001.

[5] J.-K. Chen and J.-L. C. Wu, "Heuristic batching policies for video-on-demand services", Computer Communications, Vol.22, pp.1198-1205, 1999.

[6] A. Dan, D. Sitaram, and P. Shahabuddin, "Dynamic batching policies for an on-demand video server", Multimedia Systems, Vol.4, pp.112-121, June 1996.

[7] D. Eager and J. Z. M. Vernon, "Bandwidth Skimming: A Technique for Cost-Effective Video-on-Demand", Proc. Multimedia Computing and Networking 2000, San Jose, CA, January 2000.

[8] L. Gao and D. Towsely, "Threshold-based multicast for continuous media delivery", IEEE Transactions on Multimedia, Vol.3, No.4, pp.405-414, December 2001.

[9] S.-H. G. Chan and F. Tobagi, "Tradeoff between System Profit and User Delay/Loss in Providing Near Video-on-Demand Service", IEEE Transactions on Circuits and Systems for Video Technology, Vol.11, No.8, Aug. 2001.

[10] K. A. Hua, Y. Cai, and S. Sheu, "Patching: A multicast technique for true video-ondemand services", Proc. of ACM Multimedia, Sept. 1998.

[11] H. Shachnai and P. Yu, "Exploring Wait Tolerance in Effective Batching for Video-onDemand Scheduling", Multimedia Systems, Vol.6, No.6, pp.382-394, 1998.

[12] S. Viswanathan and T. I. Metropolitan, "Video-on-demand service using pyramid broadcasting”, Multimedia Systems, Vol.4, No.4, pp.197-208, Aug. 1996. 